\title{
Environmentally evaluated HPLC-ELSD method to monitor enzymatic synthesis of a non-ionic surfactant
}

\author{
Yasser Gaber ${ }^{1}$, Cecilia Orellana Åkerman ${ }^{2}$ and Rajni Hatti-Kaul ${ }^{2^{*}}$
}

\begin{abstract}
Background: N-Lauroyl-N-methylglucamide is a biodegradable surfactant derived from renewable resources. In an earlier study, we presented an enzymatic solvent-free method for synthesis of this compound. In the present report, the HPLC method developed to follow the reaction between lauric acid/methyl laurate and N-methyl glucamine (MEG) and its environmental assessment are described.

Results: Use of ultraviolet (UV) absorption or refractive index (RI) detectors did not allow the detection of N-methyl glucamine (MEG). With Evaporative light scattering detector ELSD, it was possible to apply a gradient elution, and detect MEG with a limit of detection, $L O D=0.12 \mu \mathrm{g}$. A good separation of the peaks: MEG, lauric acid, product (amide) and by-product (amide-ester) was achieved with the gradient program with a run time of $40 \mathrm{~min}$. The setting of ELSD detector was optimized using methyl laurate as the analyte. LC-MS/MS was used to confirm the amide and amide-ester peaks. We evaluated the greenness of the developed method using the freely available software HPLC-Environmental Assessment Tool (HPLC-EAT) and the method got a scoring of 73 HPLC-EAT units, implying that the analytical procedure was more environmentally benign compared to some other methods reported in literature whose HPLC-EAT values scored up to 182.
\end{abstract}

Conclusion: Use of ELSD detector allowed the detection and quantification of the substrates and the reaction products of enzymatic synthesis of the surfactant, N-lauroyl-N-methylglucamide. The developed HPLC method has acceptable environmental profile based on HPLC-EAT evaluation.

Keywords: Green analytical chemistry, Chromatographic separation, Evaporative light scattering detector, Non-ionic surfactant, Mass spectrometry, HPLC-EAT

\section{Background}

$\mathrm{N}$-Acyl-N-methyl glucamides, also referred to as fatty acid glucamides or alkyl glucamides (AGs), are non-ionic surfactants derived from glucose and fatty acids. They are regarded as green chemicals due to their renewable origin, biodegradability, and low environmental impact. The chemical structure of the AGs contains an amide bond between the hydrophobic and the hydrophilic moieties (Scheme 1), which renders the molecule resistant to the alkaline conditions, a desirable property in surfactants intended for detergent applications. In addition to stability, safety, compatibility and synergism with other surfactants, AGs were used in

\footnotetext{
* Correspondence: Rajni.Hatti-Kaul@biotek.lu.se

${ }^{2}$ Department of Biotechnology, Center for Chemistry and Chemical Engineering, Lund University, P.O. Box 124, SE-221 00 Lund, Sweden Full list of author information is available at the end of the article
}

the formulations of detergent, personal care, and pharmaceutical products $[1,2]$.

Industrial synthesis of fatty acid glucamides involves a two-step reaction: glucose reacts with methylamine in the first step catalysed by Raney nickel to give $\mathrm{N}$-methyl glucamine (MEG), which then reacts with a fatty acid methyl ester to give the fatty acid $\mathrm{N}$-methyl glucamide (amide). Fatty acid glucamides can also be synthesised enzymatically using lipases as catalysts [3,4]. The enzymatic synthesis of AGs using MEG and fatty acid or fatty acid methyl ester yields a mixture of amide (surfactant) and amide-ester as a by-product (see Scheme 1) [5]. The unreacted MEG is an undesirable component in the final product, as it can be a precursor for nitrosamine, a potential carcinogen [6].
() ChemistryCentral 


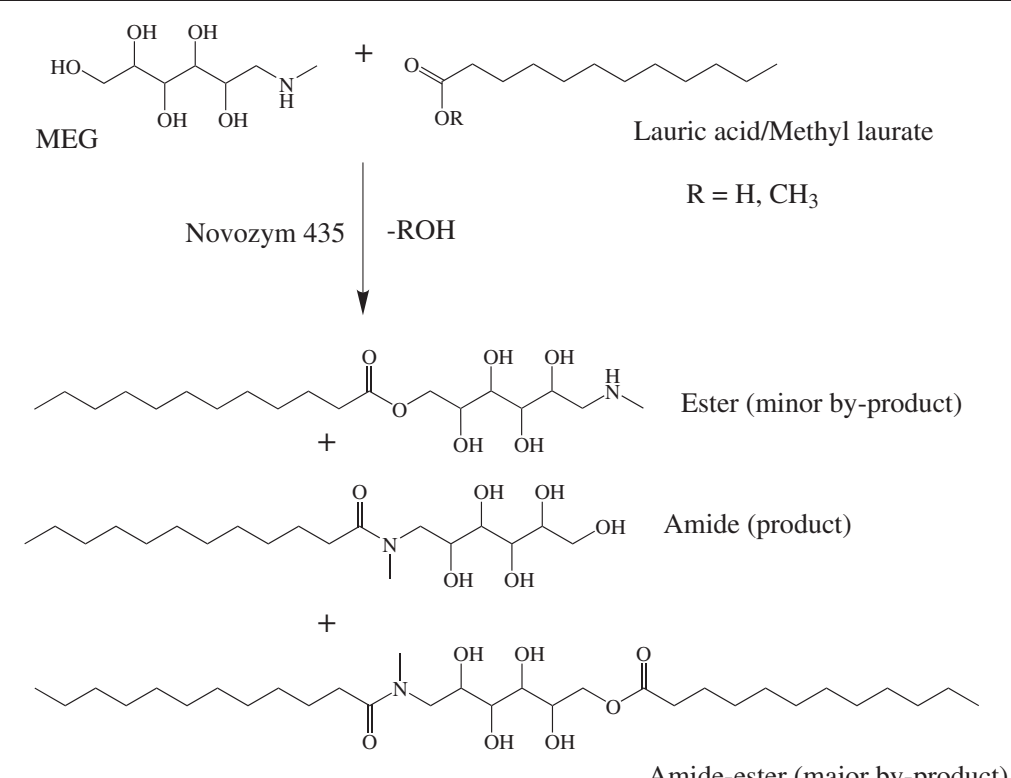

Scheme 1 Lipase-mediated synthesis of N-lauroyl-N-methyl glucamide.

A sensitive, simple, and validated analytical method is thus required to monitor the reaction and its components, especially the MEG content. GC analysis is not suitable since MEG has high melting temperature and boiling point $\left(130^{\circ} \mathrm{C}\right.$ and $490^{\circ} \mathrm{C}$, respectively). LC-MSbased analytical method has been used for monitoring the AGs in environmental and biodegradation studies [7,8]. A rapid HPLC method with UV detection or refractive index (RI) detection has also been reported [9], however in our experiments, we could not detect MEG using either UV and/or RI detectors.

In the present paper, we describe the development of an HPLC method to monitor the enzymatic synthesis of $\mathrm{N}$-lauroyl-N-methyl glucamide, and we show that ELSD was a superior alternative to UV and RI detection. The method has been validated and the safety, health and environmental impacts of the chromatographic method were assessed using the HPLC-EAT tool, the environmentalassessment software developed earlier by us for evaluating the greenness of the HPLC methods [10].

\section{Results and discussion}

\section{Reaction and detector selection}

In order to develop an enzymatic solvent-free reaction for the synthesis of N-lauroyl-N-methyl glucamide, lauric acid and/or methyl laurate were used as acyl donors or the hydrophobic component of the surfactant, and MEG was used as the acyl acceptor or the hydrophilic part. The use of lauric acid and methyl laurate alone with MEG in 1:1 molar ratio did not provide good reaction conditions; the former produced a highly viscous homogenous salt-complex while the latter led to the precipitation of MEG at the start of the reaction. Hence, lauric acid was required to keep MEG in solution, and methyl laurate lowered the viscosity and avoided the use of organic solvents in the reaction medium while also serving as acyl donor. Optimization of the reaction was based on variation of the molar ratio of lauric acid and/or methyl laurate to MEG [4]; the latter was always set to be the limiting substrate in the reaction (see the section: Monitoring the synthesis of N-lauroyl-N-methyl glucamide).

To monitor the formation of the amide in this reaction using a HPLC method, the challenge is to separate compounds that are differing in polarity, i.e. MEG is highly polar, lauric acid and amide-ester are highly nonpolar while amide has intermediate polarity.

A reaction mixture sample containing the substrates, amide and amide-ester, was chromatographed on a reverse phase column and analysed using three different detectors: UV, IR, and ELSD. Isocratic elution program (methanol: water: TFA, 75:25:0.03 v/v) was adopted with UV and RI detectors, while a gradient elution (Table 1) was applied in case of ELSD. A comparison of chromatograms obtained using the three detectors is shown in Figure 1. Two of the four analytes were detected using the UV detector, as seen in Figure 1A. The absorbance of saturated fatty acid or fatty acid methyl esters arises mainly from the carbonyl group, which accounts for the poor response in UV detection. MEG did not absorb and amide-ester was not eluted during the run time and consequently not detected.

$\mathrm{RI}$ is a universal detector, but its main limitation is that it cannot be combined with gradient elution. Isocratic elution certainly prolongs the analysis time, especially for 
Table 1 Chromatographic conditions adopted for gradient HPLC separation of reaction components in the enzymatic synthesis of $\mathrm{N}$-lauroyl $\mathrm{N}$-methyl glucamide

\begin{tabular}{|c|c|c|c|}
\hline \multirow[t]{2}{*}{ Mobile phase } & Solvent $\mathrm{A}=$ & \multicolumn{2}{|c|}{ Water: trifluoroacetic acid $(0.05 \% \mathrm{w} / \mathrm{w})$} \\
\hline & Solvent $B=$ & \multicolumn{2}{|l|}{ Methanol } \\
\hline \multirow[t]{7}{*}{ Gradient } & Time (min) & $\%$ Solvent A & $\%$ Solvent B \\
\hline & 0 & 25 & 75 \\
\hline & 5 & 25 & 75 \\
\hline & 10 & 5 & 95 \\
\hline & 10 & 5 & 95 \\
\hline & 5 & 25 & 75 \\
\hline & 10 & 25 & 75 \\
\hline Flow rate & \multicolumn{3}{|l|}{$1 \mathrm{ml} / \mathrm{min}$} \\
\hline Column & \multicolumn{3}{|c|}{ LiChrospher ${ }^{\circledast} 100$ RP-18 (5 um) (LiChroCART ${ }^{\circledast}$ 125-4 HPLC cartridge) Merck, Darmstadt, Germany } \\
\hline Column temperature & \multicolumn{3}{|l|}{$40^{\circ} \mathrm{C}$} \\
\hline Detection & \multicolumn{3}{|c|}{ ELSD (Alltech 3300, Alltech Associates, USA) } \\
\hline Injection volume & \multicolumn{3}{|l|}{$5 \mathrm{ul}$} \\
\hline \multicolumn{4}{|l|}{ ELSD settings } \\
\hline Temperature & \multicolumn{3}{|l|}{$38^{\circ} \mathrm{C}$} \\
\hline Gas flow (air) & \multicolumn{3}{|l|}{$1.3 \mathrm{~L} / \mathrm{min}$} \\
\hline Gain & \multicolumn{3}{|l|}{1} \\
\hline Run time & \multicolumn{3}{|l|}{$40 \mathrm{~min}$} \\
\hline
\end{tabular}

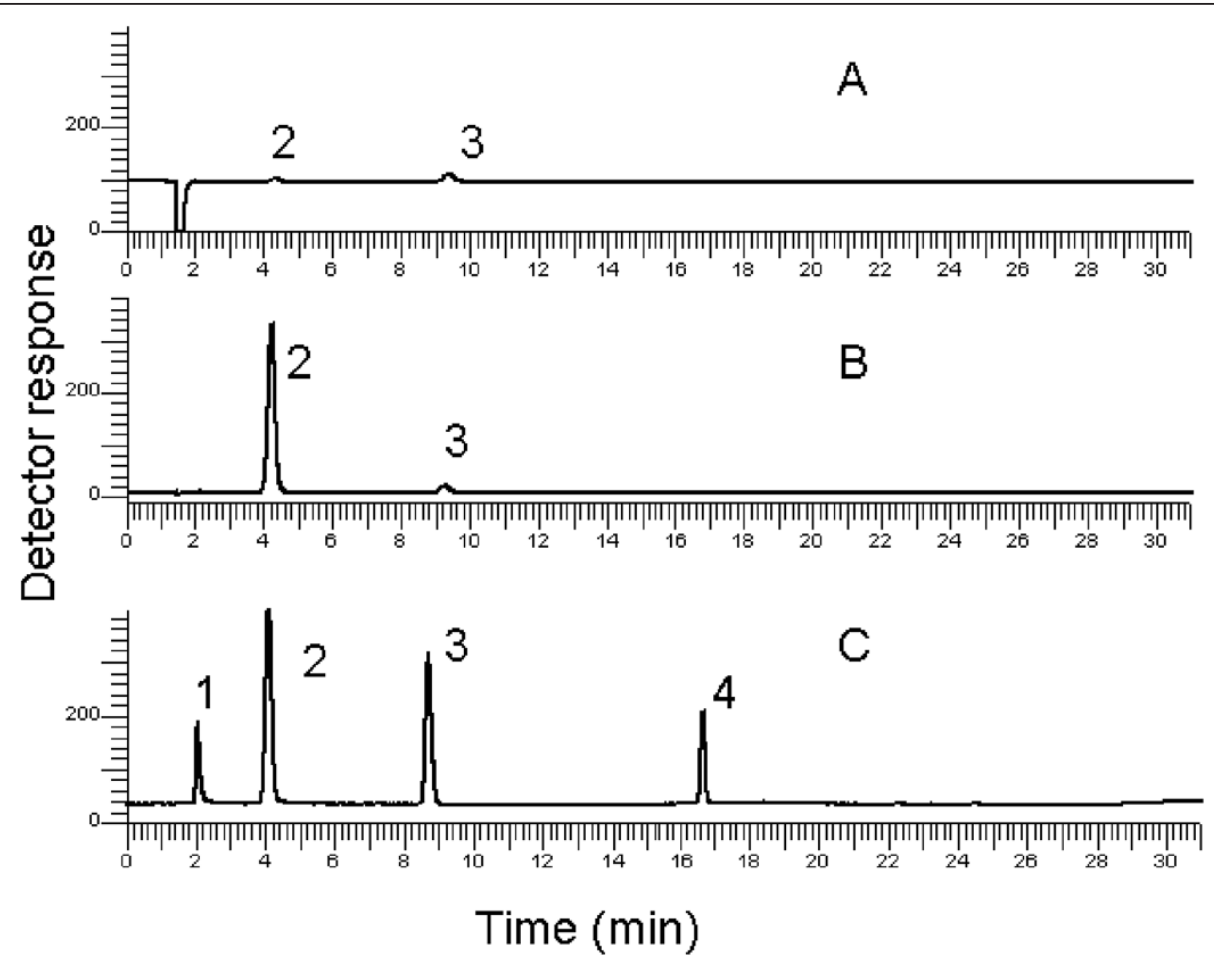

Figure 1 Comparison of HPLC analyses of the reaction components in a crude reaction mixture from the synthesis of $\mathrm{N}$-lauroyl-Nmethyl-glucamide by isocratic method using UV (A) and RI (B) detectors, and the developed gradient method using Evaporative Light Scattering Detector ELSD (C). Solutes: MEG: 1; amide: 2; lauric acid: 3; amide-ester: 4. Isocratic method: mobile phase: methanol: water: TFA

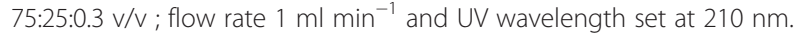



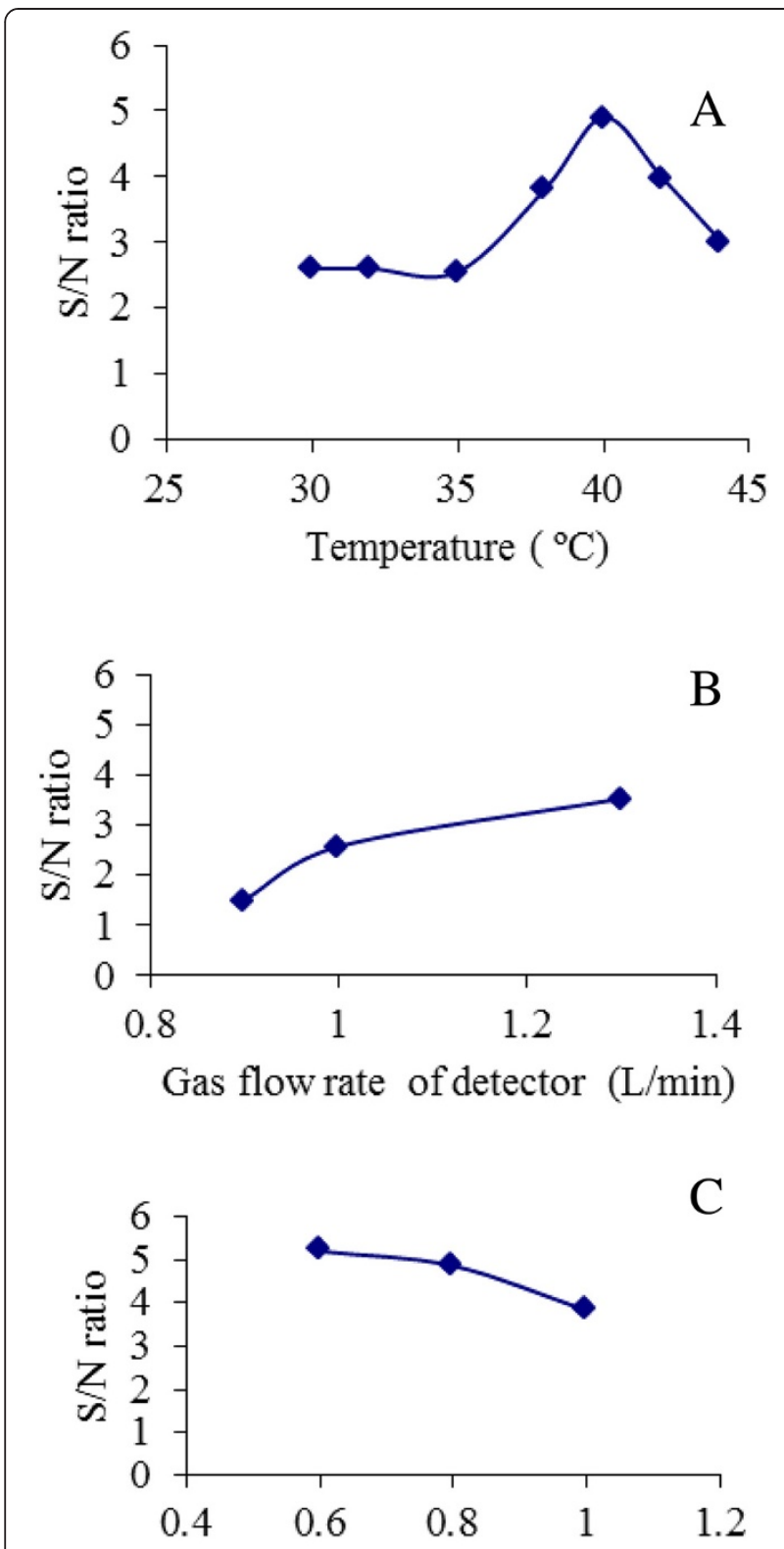

\section{Flow rate of mobile phase $(\mathrm{ml} / \mathrm{min})$}

Figure 2 ELSD optimisation for HPLC analysis of solutes. Methyl laureate was used as analyte for the optimization experiment. The Signal over Noise ratio $(S / N)$ was monitored regarding the following factors: the detector temperature $(\mathbf{A})$, the gas flow rate in the detector (B), and the flow rate of the mobile phase (C). Standard conditions are mobile phase flow rate of $1 \mathrm{ml} / \mathrm{min}, 1.3 \mathrm{~L} / \mathrm{min}$ gas flow to the detector, detector temperature is $38^{\circ} \mathrm{C}$, and gain of the detector is set to 1 .

compounds that are strongly adsorbed to the column. RI detector could only detect two of the four analytes present in the mixture: the amide and the fatty acid (Figure 1B). Notably, the detector response was very weak for the fatty acid, and it was not possible to detect MEG and amide- ester peaks. MEG has probably the same refractive index as the mobile phase. Since the amide-ester with two fatty acid moieties was very strongly adsorbed to the column, it could not be detected (Figure 1B). ELSD allowed the identification of the four analytes (Figure 1C), enabling a shorter run time and faster analysis. This is due to the possibility of gradient elution, and the absence of interference of the solvent front peak.

\section{Optimisation of the ELSD settings}

The signal intensity of analyte peaks observed by ELSD detector is highly dependent on different factors including the chromatographic conditions such as the flow rate, mobile phase composition, and settings of the detector itself. To optimize these conditions, methyl laurate was chosen as the analyte for optimization of the ELSD settings as it is the most volatile and most difficult to detect among all the analytes. Three parameters were varied: detector temperature, gas flow rate (nebulizer), and mobile phase flow rate (See Figure 2), and their effect on the Signal/Noise $(\mathrm{S} / \mathrm{N})$ ratio was monitored. S/N ratio has to be higher than 3 or 5 for detection or quantification purposes. As seen in Figure 2, temperature of the detector had a substantial effect on $\mathrm{S} / \mathrm{N}$ ratio. The detector temperature directly affected the volatilization of the carrier mobile phase solvent. The temperature settings of 38 and $40^{\circ} \mathrm{C}$ showed good $\mathrm{S} / \mathrm{N}$ ratio for methyl laurate detection (Figure 2A). The effect of pumping the filtered air into the detector at three flow rates was tested on the $\mathrm{S} / \mathrm{N}$ ratio, and $1.3 \mathrm{~L} / \mathrm{min}$ was chosen as the optimum for the detection (Figure 2B). The flow rate of the mobile phase was observed to have a significant effect on the $\mathrm{S} / \mathrm{N}$, low flow rates allowed good $\mathrm{S} / \mathrm{N}$ ratio at the expense of the elution time (Figure 2C). The final mobile phase flow rate chosen was $1 \mathrm{ml} / \mathrm{min}$ as it gave a good compromise between $\mathrm{S} / \mathrm{N}$ ratio and the overall chromatographic run time.

\section{Optimisation of the chromatographic conditions}

To obtain optimum separation between the peaks of MEG, lauric acid, amide and amide-ester, different parameters, i.e., mobile phase composition, $\mathrm{pH}$, and length of the chromatographic column, were studied. Various mobile phases with different methanol concentrations (90\%, $80 \%, 75 \%$ and $70 \% \mathrm{v} / \mathrm{v})$ were tested. Mobile phase containing 75\% methanol and LiChrospher ${ }^{\circ} 100$ RP-18, $150 \mathrm{~mm}$ column were finally chosen. The amide product could be easily separated from the ester formed as a minor product (Scheme 1) inspite of similarity in molecular weight, structure and adsorption properties. However, amide-ester was better eluted using $95 \% \mathrm{v} / \mathrm{v}$ methanol. Hence, using a methanol gradient allows the separation of all the components. A satisfactory chromatographic profile for analysis of the reaction mixture of amide surfactant 
Table 2 Calibration of the ELSD response of the four analytes based on the relationship of logarithmic values of both peak areas and analyte concentrations

\begin{tabular}{|c|c|c|c|c|c|c|c|c|c|}
\hline \multirow[t]{2}{*}{ Peak no. } & \multirow[t]{2}{*}{ Compound } & \multirow[t]{2}{*}{ Rt } & \multirow[t]{2}{*}{ Equation } & \multirow[t]{2}{*}{$\mathrm{R}^{2}$} & \multirow[t]{2}{*}{ LOD $(\mu \mathrm{g})$} & \multirow[t]{2}{*}{ LOQ $(\mu \mathrm{g})$} & \multirow[t]{2}{*}{ Linear range } & \multicolumn{2}{|c|}{ Precision (R.S.D.\%, $n=5$ ) } \\
\hline & & & & & & & & Intra-day & Inter-day \\
\hline 1 & MEG & $2.18 \pm 0.14$ & $y=1.4507 x+2.3077$ & 0.9959 & 0.12 & 0.49 & $0.49-6.20$ & 4.01 & 3.25 \\
\hline 2 & Amide & $4.31 \pm 0.30$ & $y=1.3716 x+2.3961$ & 0.9986 & 0.10 & 0.59 & $0.59-5.90$ & 2.54 & 2.24 \\
\hline 3 & Lauric & $9.15 \pm 0.48$ & $y=2.1981 x-1.5648$ & 0.9846 & 2.02 & 5.25 & $5.25-26.23$ & 3.62 & 2.98 \\
\hline 4 & Amide ester & $17.04 \pm 0.35$ & $y=1.5903 x+1.4929$ & 0.9987 & 0.04 & 0.11 & $0.11-4.52$ & 2.07 & 1.95 \\
\hline
\end{tabular}

synthesis was obtained with ELSD detector (Figure 1C). In the design of the gradient mode, we chose a mobile phase composition of $75 \%$ methanol in the first 5 minutes of the run to allow sufficient peak resolution of the amide and the ester peaks, and subsequently a gradient with increasing methanol content up to $95 \%$ was set over 10 minutes followed by holding for another ten minutes (Table 1). During the gradient run, peak 4 (amide-ester) eluted at retention time of $c a .17 .00$ minutes.

\section{Calibration curves}

The response of the ELSD detector is known to be nonlinear. Logarithmic (base 10) transformation of the analyte amount and the ELSD response was described to obtain a linear relationship $[11,12]$. Polynomial curve fitting of the ELSD detector responses was also reported; quadratic and third degree equations were described for the curve fitting with ELSD detectors [13].

Table 2 shows the calibration equations for the four analytes based on correlation between the logarithm of peak areas and the logarithm of concentrations and the corresponding $\mathrm{R}^{2}$ values. In case of MEG the second order polynomial equation (not shown in Table 2) and log-log plotting showed good $\mathrm{R}^{2}$ values 0.999 and 0.9959 , respectively. The limits of detection and quantification were based on the ratio of Signal/Noise. $S / N=3$ and $S / N=10$ respectively, were calculated for MEG, amide and amideester (see Table 2). MEG could be detected starting from

Table 3 Major ions observed by positive ESI-MS of amide and amide-ester peaks and the major fragments detected by LC-MS-MS of these two compounds

\begin{tabular}{lll}
\hline Compound & $\mathbf{~ m / z}$ & Major fragments \\
\hline Amide $\left(\mathbf{M}=\mathbf{C}_{\mathbf{1 9}} \mathrm{H}_{\mathbf{3 9}} \mathbf{N O}_{\mathbf{6}}\right)$ & 377 & \\
{$[\mathrm{M}+\mathrm{H}]^{+}$} & 378 & $360,196,78$ \\
{$[\mathrm{M}+\mathrm{Na}]^{+}$} & 400 & 382 \\
{$\left[\mathrm{M}+\mathrm{H}-\mathrm{H}_{2} \mathrm{O}\right]^{+}$} & 360 & 178,164 \\
Amide-ester $\left(\mathbf{M}=\mathbf{C}_{\mathbf{3 1}} \mathbf{H}_{\mathbf{6 1}} \mathbf{N O}_{\mathbf{7}}\right)$ & 559 & \\
{$[\mathrm{M}+\mathrm{H}]^{+}$} & 560 & $360,542,178$ \\
{$[\mathrm{M}+\mathrm{Na}]^{+}$} & 582 & \\
{$\left[\mathrm{M}+\mathrm{H}-\mathrm{H}_{2} \mathrm{O}\right]^{+}$} & 542 & 360 \\
{$[2 \mathrm{M}+\mathrm{Na}]^{+}$} & 1141 & 582 \\
\hline
\end{tabular}

$0.12 \mu \mathrm{g}$, which reflects the sensitivity of the method to monitor this critical compound in the final product. The detection limit of the amide $(0.1 \mu \mathrm{g}$ equivalent to $20 \mu \mathrm{g} / \mathrm{ml}$ ) was slightly higher than the previously reported values obtained with related non-ionic surfactants [14].

The precision of the analytical method was determined using intra- and inter-day variability measurements. Solutions of a defined concentration of reference compounds were tested. For intra-day variability, the samples were examined in triplicates three times within 1 day, while for inter-day variability, the samples were analysed in triplicates for consecutive 3 days. The obtained relative standard deviations were less than 5\% (Table 2).

\section{LC-MS identification of the unknown compounds}

Liquid Chromatography Electrospray Ionisation (LC ESI) was used to confirm the detected HPLC peaks 3 and 4 (Figure 1C). The mass spectra showed formation of sodium adduct ion in positive ion detection (Table 3). The amide and amide-ester molecules do not contain acidic or basic functional groups, and thus association with other ions in the solution is expected under electrospray ionization conditions. In the positive mode ionization mode, the amide and amide-ester compounds showed an abundant $[\mathrm{M}+\mathrm{H}]^{+}$ ion accompanied by small intensity of loss of water peak $\left[\mathrm{M}-\mathrm{H}_{2} \mathrm{O}\right]^{+}$which was also noticed as a major fragment in the collision induced dissociation step. Figure 3 shows the LC-MS-MS applied in positive ionization mode for the amide-ester. The obtained fragmentation patterns were highly consistent with their chemical structures (Table 3). These findings are in agreement with the results of Gonzalez et al. and Eichhorn et al. [7,8].

\section{Environmental assessment using HPLC-EAT software}

The developed method was evaluated further with respect to environmental impact using HPLC-EAT (Environmental Assessment Tool), an easy-to-use software providing an indication about the environmental, health and safety impacts of the chromatographic method [10]. HPLC-EAT calculates the impact of the solvents used in the analytical method and presents an output score reflecting the greenness of the method. The higher the score the less green is the method. The tool has been successfully used for evaluation of different analytical as well as preparative 


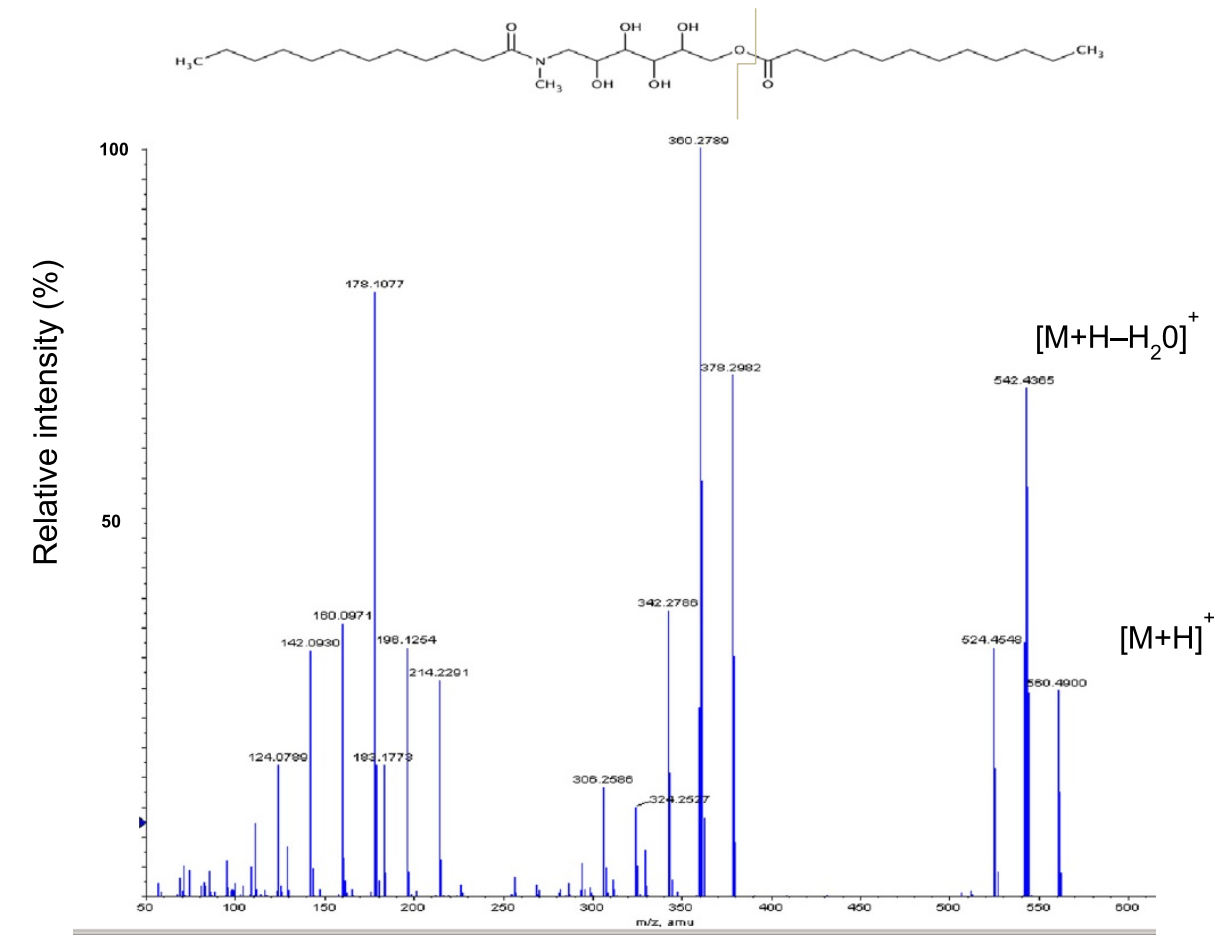

Figure 3 Positive mode LC-MS-MS chromatogram for the confirmation of amide-ester $(M w=559)$.

chromatographic methods. An advantage of HPLC-EAT over the other software package EATOS, is that it has a built-in data on risk parameters of organic solvents to do the assessments and allows reproducible assessment results [10].

For the HPLC method developed in this report, HPLCEAT gave a score of 73 HPLC-EAT units. Methanol represented $36 \mathrm{ml}$ of the $40 \mathrm{ml}$ mobile phase consumed during the chromatographic run, and this solvent amount contributed for the safety, health and environmental impact shown in Figure 4. The bar chart shows that the safety impact of the method is a major contribution to the overall impact that is related to methanol being a flammable solvent. HPLC-EAT does not assign penalties to use of water. In our previous study, HPLC-EAT of different analytical methods developed to analyse different types

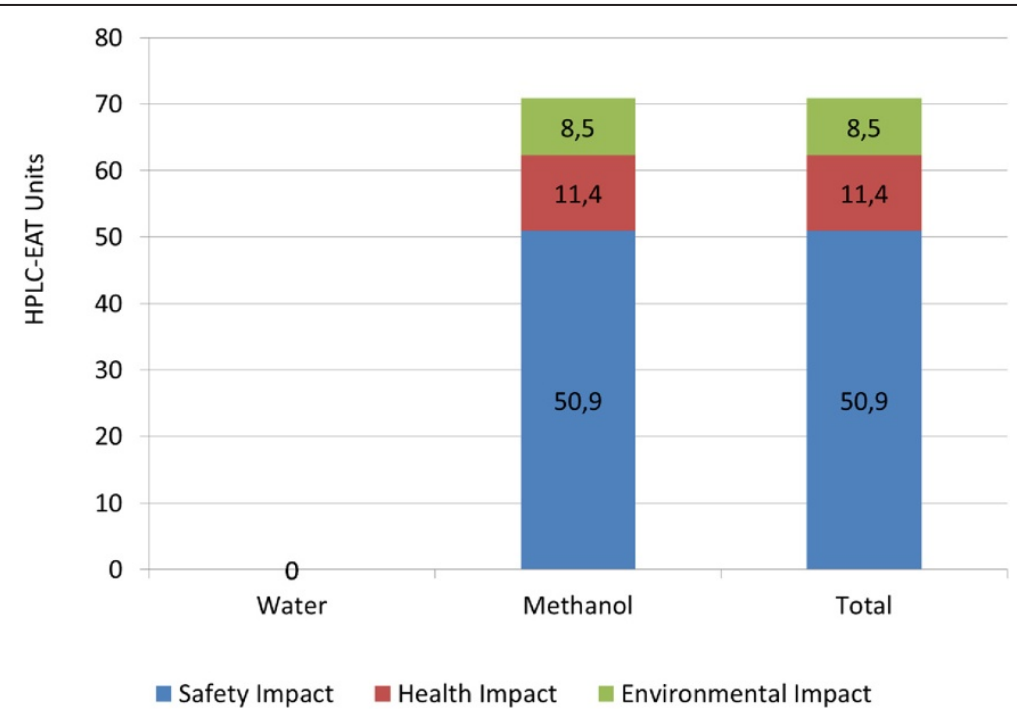

Figure 4 Evaluation of environmental, health and safety impact of the chromatographic method described in Table 1 using HPLC-EAT software. 
of surfactants showed a score ranging from to 43 to 182 [10]. Based on this comparison, we can state that our method is environmentally acceptable and lies within the HPLC-EAT score range of HPLC methods described for surfactants analysis.

Monitoring the synthesis of $\mathrm{N}$-lauroyl-N methyl glucamide The HPLC method described here was successfully used to quantify the enzymatic synthesis of N-lauroyl-N methyl glucamide. The method allowed good separation and quantification of main reaction analytes i.e., the limiting substrate (MEG), the product (amide) and the by-product (amide-ester). Different molar ratios of MEG, lauric acid and/or methyl laurate were mixed and the reactions were catalysed by an immobilized lipase preparation. When lauric acid and methyl laurate were used together as substrates, the methyl laurate content in the reaction was rapidly decreased after the start of the reaction (Figure $1 \mathrm{C}$ ). This is due to the lipase mediated hydrolysis of methyl laurate to lauric acid even under the solvent free conditions and is noted as the disappearance of methyl laurate peak in Figure 1C. A solvent-free reaction including lauric acid, methyl laurate and MEG in a 1:1:1 molar ratio resulted in N-lauroyl-N methyl glucamide yield of $34 \%$ with amide-ester as a major by-product. The final reaction mixture was subjected to hydrolysis under mild alkaline conditions to give a final amide yield of $99 \%$ and the excess acylating agent remained as laurate that can be recycled for subsequent reactions. The HPLC method allowed the monitoring of the reaction time course since MEG is the limiting substrate of the reaction. It could also confirm the full conversion of MEG since it showed high sensitivity $(\mathrm{LOD}=0.12 \mu \mathrm{g})$.

\section{Materials and methods Reagents and chemicals}

Novozym $^{\circ} 435$ (immobilised lipase from Candida antarctica of 10,000 Propyl Laurate Units (PLU) per gram), was a gift from Novozymes (Bagsvaerd, Denmark). N-methylglucamine (MEG) was purchased from Sigma. HPLCgrade methanol, lauric acid and trifluoroacetic acid for spectroscopy were purchased from Merck. Methyl laurate was procured from Fluka. Milli-Q (Millipore, Milford, MA, USA) quality water was used. N-Lauroyl-N-methyl glucamide (amide) and O-lauroyl-N-lauroyl methyl glucamide (amide-ester) were produced in-house enzymatically and purified using flash chromatography according to Maugard et al. [15]. Structure confirmation was done using infrared and mass spectroscopy.

\section{HPLC apparatus and chromatographic conditions}

HPLC from PerkinElmer Series 200 system equipped with a binary pump, autosampler, oven, interface (NCI 900), and three detectors: UV from PerkinElmer 785A, RI from
Hitachi L-2490 and ELSD from Alltech (3300, Alltech Associates, USA), was used. ELSD was operated in a temperature range of $25^{\circ} \mathrm{C}$ to $45^{\circ} \mathrm{C}$ and a gas flow of $1.3 \mathrm{~L} /$ min and gain of 1 . N-Methyl glucamide, fatty acid and their products were separated on an LiChrospher 100 RP-18 HPLC column, with a guard cartridge RP-18 from Merck, Darmstadt, Germany. Aqueous solution of methanol was used as mobile phase and the injection volume was $5 \mu \mathrm{l}$.

\section{Enzymatic synthesis of $\mathrm{N}$-methyl-N-lauroyl glucamine}

$\mathrm{N}$-Methyl glucamine $(3.5 \mathrm{mmol})$ was mixed with lauric acid $(3.5 \mathrm{mmol})$ and methyl laurate $(3.5 \mathrm{mmol}$ total $)$ in a round bottom flask, and the reaction was run in solventfree medium under stirring at $90^{\circ} \mathrm{C}$. Novozym ${ }^{\circ} 435$ at $4 \%$ $\mathrm{w} / \mathrm{w}$ of total substrates weight was added as catalyst in all reactions. The details of the reaction were reported elsewhere [4].

\section{Mass spectrometry}

Mass spectrometry of O-lauroyl-N-lauroyl methyl glucamide (amide-ester) was conducted on a hybrid QS-STAR Pulsar quadrupole TOF mass spectrometer (PE Sciex Instruments, Toronto, Canada). The spectrometer was connected with a similar LC-HPLC system. The electrospray ionization (ESI) source was set to positive ion mode. The quadrupole system was adjusted to scan between $\mathrm{m} / \mathrm{z}$ 100-2000 in TOF-MS mode whereas for product ion mode (i.e., MS/MS) a range of $\mathrm{m} / \mathrm{z}$ 50-2000 was chosen. Data was assessed using the Analyst ${ }^{\oplus}$ QS software (PE Sciex Instruments, Toronto, Canada).

\section{Conclusion}

Monitoring the enzymatic synthesis of the surfactant N-lauroyl-N-methylglucamide was achieved by a HPLC method with ELSD. This method is a better alternative to the previously reported HPLC method using UV or RI detectors. It was very sensitive for detecting MEG (LOD = $0.12 \mu \mathrm{g}$ ), which enables the detection of trace amounts of the compound in the final surfactant product. Calibration curves of the different analytes using ELSD as detector were made using double-logarithmic (log-log) relation. The greenness profile of the method was evaluated using HPLC-EAT software and was found to be acceptable. The method was successfully used to monitor solvent-free synthesis of the surfactant, which is free from the substrate MEG.

\section{Abbreviations}

AGs: Alkyl glucamides; MEG: N-methyl glucamine; Amide: N-lauroyl-N-methyl glucamide; TFA: Trifluoroacetic acid; UV: Ultra-violet; RI: Refractive index; ELSD: Evaporative light scattering detector; LOD: Limit of detection; LOQ: Limit of quantification; TOF: Time of flight; S/N: Signal over noise ratio; HPLC-EAT: HPLC-environmental assessment tool. 


\section{Competing interests}

The authors declare that they have no competing interests.

\section{Authors' contributions}

YG did the experiments and wrote the manuscript, $C O \AA$, supervised the experiments and suggested use of ELSD and discussed the results, RHK supervised the whole project and revised the manuscript. All the authors read and approved the final manuscript.

\section{Acknowledgment}

Egyptian Ministry of Higher Education and Beni-Suef University, Egypt are acknowledged for supporting YG.

\section{Author details}

${ }^{1}$ Department of Microbiology, Faculty of Pharmacy, Beni-Suef University, Salah Salem street, 62511 Beni-Suef, Egypt. 'Department of Biotechnology, Center for Chemistry and Chemical Engineering, Lund University, P.O. Box 124, SE-221 00 Lund, Sweden.

Received: 4 March 2014 Accepted: 15 May 2014

Published: 20 May 2014

\section{References}

1. Steckel H, Eskandar F, Witthohn $K$ : The effect of formulation variables on the stability of nebulized aviscumine. Int J Pharm 2003, 257(1-2):181-194.

2. Naik A: Detergent formulations based on soap and fatty acid N-methyl glucamide. 2007, WO Patent 2,007,017,619.

3. Masyithah Z, Sembiring SB, Alfian Z, Herawan T: The optimization of enzymatic synthesis for lauroyl-n-methyl glucamide surfactants. Indonesian J Chem 2011, 11(3):223-228.

4. Gaber Y, Törnvall U, Orellana-Coca C, Ali Amin M, Hatti-Kaul R: Enzymatic synthesis of $\mathrm{N}$-alkanoyl-N-methylglucamide surfactants: solvent-free production and environmental assessment. Green Chem 2010, 12(10):1817-1825.

5. Maugard T, Remaud-Simeon M, Petre D, Monsan P: Enzymatic amidification for the synthesis of biodegradable surfactants: synthesis of N-acylated hydroxylated amines. J Mol Catal B Enzym 1998, 5(1-4):13-17.

6. Hill K, Rhode O: Sugar-based surfactants for consumer products and technical applications. Fett-Lipid 1999, 101(1):25-33.

7. González S, Barceló D, Petrovic M: Advanced liquid chromatography-mass spectrometry (LC-MS) methods applied to wastewater removal and the fate of surfactants in the environment. Trends Anal Chem 2007, 26(2):116-124.

8. Eichhorn P, Knepper T: Fate studies of the non-ionic surfactant alkyl glucamide by liquid chromatography/electrospray mass spectrometry. J Mass Spectrom 2000, 35(3):468-475.

9. Maugard T, Remaud-Simeon M, Petre D, Monsan P: Lipase-catalysed synthesis of biosurfactants by transacylation of N-methyl-glucamine and fatty-acid methyl esters. Tetrahedron 1997, 53(22):7629-7634.

10. Gaber Y, Törnvall U, Kumar MA, Ali Amin M, Hatti-Kaul R: HPLC-EAT (Environmental Assessment Tool): a tool for profiling safety, health and environmental impacts of liquid chromatography methods. Green Chem 2011, 13(8):2021-2025.

11. Wang Q, Zhang S, Yang J: HPLC analysis of sucrose ester analogs using Evaporative Light Scattering Detection. J Liq Chromatogr Relat Technol 2007, 30(16):2395-2407.

12. Aruda WO, Walfish S, Krull IS: Review and optimization of linearity and precision in quantitative HPLC-ELSD with chemometrics. LC GC North Am 2008, 26(10):1032-1042.

13. Orellana-Coca C, Adlercreutz D, Andersson MM, Mattiasson B, Hatti-Kaul R: Analysis of fatty acid epoxidation by high performance liquid chromatography coupled with evaporative light scattering detection and mass spectrometry. Chem Phys Lipids 2005, 135(2):189-199.
14. Park Hong S, Ryu Ho R, Rhee Choong K: Simultaneous separation of nine surfactants of various types by HPLC with evaporative light scattering detection. Talanta 2006, 70(3):481-484.

15. Maugard T, Remaud-Simeon M, Monsan P: Chemoselective amidification of amino-polyols catalyzed with lipases in organic solvents. Methods Biotechnol 2001, 15:325-330.

doi:10.1186/1752-153X-8-33

Cite this article as: Gaber et al:: Environmentally evaluated HPLC-ELSD method to monitor enzymatic synthesis of a non-ionic surfactant.

Chemistry Central Journal 2014 8:33.

\section{Publish with ChemistryCentral and every scientist can read your work free of charge \\ "Open access provides opportunities to our colleagues in other parts of the globe, by allowing anyone to view the content free of charge." W. Jeffery Hurst, The Hershey Company. \\ - available free of charge to the entire scientific community \\ - peer reviewed and published immediately upon acceptance \\ - cited in PubMed and archived on PubMed Central \\ - yours - you keep the copyright \\ Submit your manuscript here: \\ http://www.chemistrycentral.com/manuscript/<smiles>c1ccccc1</smiles> \\ Chemistry Central}

